\title{
In Vitro Efficacy Comparison of Linezolid, Tedizolid, Sutezolid and Delpazolid Against Rapid Growing Mycobacteria Isolated in Beijing, China
}

\section{Shuan Wen}

Beijing Chest Hospital

\section{Xiaopan Gao}

Chinese Academy of Medical Sciences \& Peking Union Medical College Institute of Pathogen Biology

\section{Weijie Zhao}

Beijing Chest Hospital

\section{Fengmin Huo}

Beijing Chest Hospital

\section{Guanglu Jiang}

Beijing chest hospital

\section{Lingling Dong}

Beijing Chest Hospital

\section{Liping Zhao}

Beijing Chest Hospital

\section{Fen Wang}

Beijing Chest Hospital

Xia Yu ( $\sim$ yuxiasmart@163.com )

Beijing Chest Hospital https://orcid.org/0000-0002-1400-7321

\section{Hairong Huang}

Beijing Chest Hospital

\section{Research}

Keywords: Rapid growing Mycobacteria, delpazolid, sutezolid, tedizolid, linezolid, antimicrobial activity

Posted Date: February 2nd, 2021

DOI: https://doi.org/10.21203/rs.3.rs-159157/v1

License: (1) (1) This work is licensed under a Creative Commons Attribution 4.0 International License.

Read Full License 


\section{Abstract}

The natural resistance of rapid growth Mycobacterium (RGM) against multiple antibiotics renders the treatment of caused infections less successful and time consuming. Therefore, new effective agents are urgently needed. The aim of this study was to evaluate the in vitro susceptibility of 115 isolates, constituting different RGM species, to four oxazolidinones i.e. delpazolid, sutezolid, tedizolid and linezolid. Additionally, 32 reference strains of different RGM species were also tested. The four oxazolidinones exhibited potent in vitro activity against the recruited RGM reference strains, 24 out of 32 RGM species had MICs $\leq 8 \mu \mathrm{g} / \mathrm{mL}$ against all four oxazolidinones whereas tedizolid and delpazolid generally presented lower MICs than linezolid or sutezolid. Tedizolid showed the strongest activity against clinical isolates of $M$. abscessus with $\mathrm{MIC}_{50}=1 \mu \mathrm{g} / \mathrm{mL}$ and $\mathrm{MIC}_{90}=2 \mu \mathrm{g} / \mathrm{mL}$. MIC values for tedizolid were usually 4- to 8-fold less than the MICs of linezolid for M. abscessus subsp. abscessus. The MIC distributions of sutezolid and linezolid were similar, while delpazolid showed 2-fold lower MIC as compared with linezolid. Linezolid was not active against most of the tested $M$. fortuitum isolates, since 22 out of the $25 \mathrm{M}$. fortuitum were resistant against linezolid. However, delpazolid exhibited better antimicrobial activity against these isolates with 4-fold lower MIC values, in contrast with linezolid. In addition, the protein alignment of RpIC and RpID and structure based analysis showed that there may be no correlation between oxazolidinones resistance and mutations in rp/C,rp/D and 23srRNA genes in tested RGM. This study showed tedizolid harbors the strongest inhibitory activity against $M$. abscessus in vitro, while delpazolid presented the best activity against $M$. fortuitum, which provided important insights on the potential clinical application of oxazolidinones to treat RGM infections.

\section{Introduction}

Non-tuberculous mycobacteria (NTM) are recognized as important opportunistic pathogens of humans that can cause pulmonary infection, lymphadenitis, skin abscesses, disseminated infection and systematic infection. The prevalence of NTM infections has increased globally and even surpassed tuberculosis (TB) in certain countries (1-5). According to their speed of growth (i.e. appearance of visible colonies within or after 7 day cultivation on solid medium), NTM can be categorized as rapid growing Mycobacteria (RGM) or slow growing mycobacteria (SGM). Compared with SGM, RGM are more resistant to conventional anti-TB agents and other general antibiotics, therefore, increasing the chances of treatment failure(6). M. abscessus and $M$. fortuitum are among the most frequently isolated and pathogenic RGMs(1-5). M. abscessus often cause severe pulmonary infections with poor clinical outcomes and have been frequently reported to cause soft tissue infections (7). M. fortuitum can cause soft tissue infection during trauma and surgery, while lung disease caused by them is rare(8). The limited efficacies and availability of only fewer choices of medications highlight the requirement of identifying new and more potent antimicrobials against RGMs.

Oxazolidinones have demonstrated promising efficacies against $M$. tuberculosis (TB) in vitro and in vivo. Due to their distinct mechanism of action (binding to the 23S ribosome, thereby blocking microbial protein synthesis) without cross-resistance to the existing TB drugs. Oxazolidinones are proposed to be 
used for the treatment of multiple drug resistant TB. Linezolid (LZD), licensed in 2000, is an oxazolidinone which exhibited excellent antibacterial activity against drug resistant tuberculosis (DR-TB) and NTM infection (9-12). However, serious hematologic and neurologic toxicities can be caused by LZD during long term therapy due to its inhibition of mitochondrial protein synthesis which often requires dose reduction or discontinuation(13). Thus, new oxazolidinones drugs with superior efficacy and reduced toxicity are continuously sought.

Recently, three new next-generation oxazolidinones have been developed for potential use against DR-TB. Tedizolid (TZD) phosphate is a novel, potent oxazolidinone pro-drug that has been approved by the American FDA (2014) and the European Medicine Agency (2015) for treatment of acute bacterial skin and soft tissue infections(7). The pharmacokinetic/pharmacodynamic properties of TZD allow it to be administered orally once daily, facilitating its usage in a prolonged treatment course. Sutezolid (SZD) (PNU-100480) is a thiomorpholinyl analog of LZD with preliminary evidence of superior efficacy against M. tuberculosis(14). SZD was found to be generally safe, well tolerated in TB patients, and with readily detectable bactericidal activity in sputum and blood. Delpazolid (LCB01-0371) (DZD) is a thiomorpholinyl analog of LZD, which showed superior efficacy against $M$. tuberculosis in the hollow-fiber, mouse model, and whole-blood model (2-4). DZD was well tolerated and showed bacteriostatic and bactericidal activity comparable to LZD against $S$. aureus, E. faecalis and methicillin-resistant Staphylococcus aureus in a recently completed phase I clinical trial(15).

To better understand the efficacies of these three new-generation oxazolidinones against different RGM species, we determined the MICs of 32 RGM reference strains and 115 RGM clinical isolates collected in Beijing, China. Furthermore, we investigated the three reported LZD-resistance genes (including $r p / C, r p / D$ and 23srRNA) from different RGM species to identify their potential relationships with oxazolidinone resistance.

\section{Results}

\section{MICs of SZD, TZD, DZD and LZD against RGM reference strains}

The MICs of the 32 reference strains against SZD, TZD, DZD and LZD are presented in Table 1. All four oxazolidinones exhibited antimicrobial activities in vitro against the recruited RGM reference stains. Majority of the species had MICs equal to or below $8 \mu \mathrm{g} / \mathrm{mL}$ for all four drugs. Only $M$. fortuitum and $M$. rhodesiae had MICs greater than $32 \mu \mathrm{g} / \mathrm{mL}$. Generally, a given isolate presented uniform tendency against all four oxazolidinones, all of the MIC values were either high or low for the four drugs. For $M$. abscessus, the efficacy of TZD was stronger than LZD. 
Table 1

MICs of LZD, TZD, SZD and DZD against the reference strains of 32 RGM species.

\begin{tabular}{|c|c|c|c|c|c|}
\hline \multirow[t]{2}{*}{ Strain by type } & \multirow[t]{2}{*}{ Mycobacterium species (strain) } & \multicolumn{4}{|c|}{$\operatorname{MIC}(\mu \mathrm{g} / \mathrm{ml})$} \\
\hline & & LZD & TZD & SZD & DZD \\
\hline ATCC 19977 & Mycobacterium abscessus & 16 & 4 & 8 & 8 \\
\hline ATCC 27406 & Mycobacterium agri & 0.25 & 0.25 & 0.25 & 0.5 \\
\hline ATCC 27280 & Mycobacterium aichiense & 0.5 & 0.5 & 2 & 1 \\
\hline ATCC 23366 & Mycobacterium aurum & 0.25 & 0.125 & 0.25 & 0.125 \\
\hline ATCC 33464 & Mycobacterium austroafricanum & 0.25 & 0.25 & 2 & 0.5 \\
\hline ATCC 14472 & Mycobacterium chelonae & 8 & 8 & 4 & 8 \\
\hline ATCC 19627 & Mycobacterium chitae & 1 & 1 & 2 & 1 \\
\hline ATCC 27278 & Mycobacterium chubuense & 32 & 2 & 2 & 2 \\
\hline DSM 44829 & Mycobacterium cosmeticum & 4 & 2 & 16 & 1 \\
\hline ATCC 19340 & Mycobacterium diernhoferi & 1 & 0.5 & 2 & 1 \\
\hline ATCC 43910 & Mycobacterium duvalii & 1 & 0.25 & 0.5 & 0.5 \\
\hline ATCC 35219 & Mycobacterium fallax & 4 & 2 & 8 & 1 \\
\hline ATCC 14474 & Mycobacterium flavescens & 16 & 2 & 2 & 8 \\
\hline ATCC 6841 & Mycobacterium fortuitum & 32 & $>32$ & $>32$ & $>32$ \\
\hline ATCC 27726 & Mycobacterium gadium & 0.5 & 0.125 & 0.25 & 0.125 \\
\hline ATCC 43909 & Mycobacterium gilvum & 0.5 & 0.25 & 0.5 & 0.5 \\
\hline ATCC BAA-955 & Mycobacterium goodii & 16 & 4 & 32 & 2 \\
\hline DSM 44124 & Mycobacterium mucogenicum & 1 & 1 & 1 & 1 \\
\hline ATCC 25795 & Mycobacterium neoaurum & 1 & 0.5 & 2 & 1 \\
\hline ATCC 27023 & Mycobacterium obuense & 0.5 & 0.25 & 0.5 & 0.5 \\
\hline ATCC 19686 & Mycobacterium parafortuitum & 1 & 0.5 & 2 & 1 \\
\hline DSM 43271 & Mycobacterium peregrinum & 2 & 4 & 4 & 1 \\
\hline ATCC 11758 & Mycobacterium phlei & 2 & 4 & 8 & 16 \\
\hline ATCC 33776 & Mycobacterium porcinum & 16 & 8 & 32 & 4 \\
\hline ATCC 35154 & Mycobacterium pulveris & 1 & 1 & 1 & 2 \\
\hline ATCC 27024 & Mycobacterium rhodesiae & $>32$ & $>32$ & $>32$ & 16 \\
\hline
\end{tabular}




\begin{tabular}{|c|c|c|c|c|c|}
\hline \multirow[t]{2}{*}{ Strain by type } & \multirow[t]{2}{*}{ Mycobacterium species (strain) } & \multicolumn{4}{|c|}{$\mathrm{MIC}(\mu \mathrm{g} / \mathrm{ml})$} \\
\hline & & LZD & TZD & SZD & DZD \\
\hline ATCC 700731 & Mycobacterium septicum & 16 & 8 & 8 & 8 \\
\hline ATCC 19420 & Mycobacterium smegmatis & 2 & 2 & 4 & 4 \\
\hline ATCC 19527 & Mycobacteriumthermoresistibile & 4 & 2 & 2 & 4 \\
\hline ATCC 27282 & Mycobacterium tokaiense & 1 & 2 & 2 & 1 \\
\hline ATCC 23292 & Mycobacterium triviale & 2 & 2 & 2 & 2 \\
\hline ATCC 15483 & Mycobacterium vaccae & 2 & 0.5 & 1 & 1 \\
\hline
\end{tabular}

The MIC distributions of $M$. abscessus and M. massiliense against LZD, TZD, SZD and DZD are shown in Fig. 1. MICs for TZD were generally 4- to 8-fold less than the MICs of LZD for the two species. The MIC distribution of SZD was similar to LZD, while DZD values were generally half of LZD. Notably, TZD showed strongest activity against $M$. abscessus with $\mathrm{MIC}_{50}=1 \mu \mathrm{g} / \mathrm{mL}$ and $\mathrm{MIC}_{90}=2 \mu \mathrm{g} / \mathrm{mL}$. According to the CLSI resistance criteria for LZD, the susceptibility rate of $M$. abscessus to LZD, TZD, SZD and DZD was $73.5 \%(36 / 49), 100 \%(49 / 49), 71.4 \%(35 / 49), 87.8 \%(43 / 49)$, respectively. The susceptibility rate of $M$. massiliense to LZD, TZD, SZD and DZD was 65.8\%(23/35), 82.9\%(29/35), 68.6\%(24/35) and 74.3\% (26/35), respectively. In general, the MIC distributions of $M$. massiliense had a more uniform tendency than M. abscessus, with an exception for TZD. The MICs of $M$. massiliense isolates were higher than $M$. abscessus. Six out of 35 isolates of $M$. massiliense had MICs $\geq 16 \mu \mathrm{g} / \mathrm{mL}$ against TZD, the MICs of all the tested $M$. abscessus were $\leq 4 \mu \mathrm{g} / \mathrm{mL}$. In addition, the MIC outcomes for species with less than five isolates are presented in Table 2.

Table 2

MICs of LZD, TZD, SZD and DZD against 6 clinical isolates of RGM species.

\begin{tabular}{|llllll|}
\hline Mycobacterium species (strain) & Clinical isolates number & \multicolumn{4}{l}{ MIC $(\mu \mathrm{g} / \mathrm{ml})$} \\
\cline { 3 - 6 } & & LZD & TZD & SZD & DZD \\
\hline Mycobacterium chelonae & 585 & 32 & 8 & 16 & 8 \\
\hline Mycobacterium chelonae & 752 & $>32$ & 16 & 8 & 16 \\
\hline Mycobacterium chelonae & 1354 & 4 & 1 & 2 & 1 \\
\hline Mycobacterium chelonae & 1392 & 4 & 1 & 2 & 1 \\
\hline Mycobacterium chelonae & 1593 & 4 & 0.5 & 2 & 0.5 \\
\hline Mycobacterium porcinum & 29891 & 4 & 2 & 4 & 1 \\
\hline
\end{tabular}




\section{The MIC distributions of M. fortuitum against LZD, TZD, SZD and DZD}

The MIC distributions of $M$. fortuitum against LZD, TZD, SZD and DZD are shown in Fig. 2. In contrast to $M$. abscessus and $M$. massiliense, $M$. fortuitum presented higher percentage of resistance against the four oxazolidinones. The susceptibility profiles of the clinical isolates were in concordance with the $M$. fortuitum ATCC6481 reference strains. In Total, 88\% (22/25) of the clinical isolates were resistant to LZD. The in vitro activity of DZD was relatively better than LZD as indicated by its 2- to 4-fold lower MIC. The MIC distributions of TZD were similar to LZD as only 5 out of 25 isolates indicated MIC $\leq 8 \mu \mathrm{g} / \mathrm{mL}$. According to the cutoff value of LZD, the susceptibility rates of $M$. fortuitum against TZD, SZD and DZD were $20 \%(5 / 25), 12 \%(3 / 25), 76 \%$ (19/25), respectively.

\section{Alternations in the Oxazolidinones target sites}

The entire 23SrRNA, rp/C, and $r p / D$ genes were sequenced to identify the potential mutations associated with oxazolidinones resistance. The sequences of the tested clinical isolates of $M$. abscessus, $M$. massiliense and $M$. fortuitum were compared with their corresponding reference strains. For $M$. massiliense isolates ,Ala177Proin $r p / D$ was detected in 12 isolates with MIC of LZD $\geq 2 \mu \mathrm{g} / \mathrm{mL}$. In addition, two types of synonymous SNPs within the coding region of $r p / C$ were also observed both in LZD resistant and susceptible isolates, including Leu86Leu(CTG $\rightarrow$ CTT) and Ala92Ala(GCG $\rightarrow$ GCT). A2271G in 23SrRNA was found in one isolate with MIC of $L Z D=8 \mu \mathrm{g} / \mathrm{mL}$. For $M$. abscessus isolates, no nonsynonymous mutation in the coding gene of $r p / C$ and $r p / D$ was observed, while most frequently observed mutation i.e. T2650C ( $n=2)$ was found in 23SrRNA with MIC of LZD $\geq 2 \mu \mathrm{g} / \mathrm{mL}$ (Table 3). 
Table 3

The MICs of LZD and rp/C, rp/D and 23srRNA mutations against $M$. abscessus and $M$. massiliense isolates.

\begin{tabular}{|c|c|c|c|c|}
\hline MIC of LZD $(\mu \mathrm{g} / \mathrm{ml})$ & Species (NO.) & RplC & RplD & 23SrRNA \\
\hline \multirow[t]{2}{*}{0.25} & M. abscessus $(0)$ & - & - & - \\
\hline & M.massiliense(1) & Leu86Leu(1) & Gly75Gly(1) & WT \\
\hline \multirow[t]{2}{*}{1} & M. abscessus(1) & WT & WT & WT \\
\hline & M.massiliense(0) & - & - & - \\
\hline \multirow[t]{5}{*}{2} & M. abscessus(4) & WT & WT & G1914A (1) \\
\hline & & & & $\mathrm{T} 2650 \mathrm{C}(1)$ \\
\hline & M.massiliense(4) & Leu86Leu(2) & Gly75Gly(2) & WT \\
\hline & & & Ala177Pro(2) & \\
\hline & & & Val192Val(2) & \\
\hline \multirow[t]{4}{*}{4} & M. abscessus(14) & WT & Phe23Phe(2) & $\mathrm{T} 2650 \mathrm{C}(1)$ \\
\hline & M.massiliense(8) & Leu86Leu(5) & Gly75Gly(5) & $\mathrm{G} 2582 \mathrm{C}(1)$ \\
\hline & & & Ala177Pro (3) & $-2625 \mathrm{AC}(1)$ \\
\hline & & & Val192Val(3) & \\
\hline \multirow[t]{6}{*}{8} & M. abscessus(17) & WT & Phe23Phe(3) & WT \\
\hline & & & Gly111Gly(1) & \\
\hline & M.massiliense(10) & Leu86Leu(4) & Ile29\|le(1) & $\mathrm{A} 2271 \mathrm{G}(1)$ \\
\hline & & Ala92Ala(2) & Gly75Gly(3) & \\
\hline & & & Ala177Pro (4) & \\
\hline & & & Val192Val(6) & \\
\hline \multirow[t]{4}{*}{16} & M. abscessus(13) & WT & Phe23Phe(3) & WT \\
\hline & M.massiliense(5) & Leu86Leu(2) & Gly75Gly(2) & WT \\
\hline & & & Ala177Pro (3) & \\
\hline & & & Val192Val(3) & \\
\hline \multirow[t]{3}{*}{$>16$} & M. abscessus(0) & - & - & - \\
\hline & M.massiliense(7) & Leu86Leu(1) & Gly75Gly(1) & WT \\
\hline & & Ala92Ala(6) & Val192Val(5) & \\
\hline
\end{tabular}


Among the tested $M$. fortuitum isolates, all MICs for LZD were above $2 \mu \mathrm{g} / \mathrm{mL}$. No non-synonymous mutation was detected in the rp/C gene. Among 25 tested $M$. fortuitum isolates, A2090T and C1944T were detected in two isolates with $\mathrm{MIC}=32 \mu \mathrm{g} / \mathrm{mL}$ and $8 \mu \mathrm{g} / \mathrm{mL}$ for LZD, respectively. In addition, 21 out of 25 clinical $M$. fortuitum isolates simultaneously showed following nine non-synonymous mutations in the coding region of $r p / D$ for the both LZD susceptible and resistant isolates: Ala146Gly(GCG $\rightarrow G G C)$, Thr147Ser(ACC $\rightarrow$ AGC), Val156lle(GTG $\rightarrow$ ATC), Ala161Thr(GCG $\rightarrow$ ACC), Lys167Arg(AAG $\rightarrow$ CGC), Ser207Ala(TCC $\rightarrow$ GCG), Glu212Gly (GAG $\rightarrow$ GGA), Val213Ala(GTG $\rightarrow$ GCG), Ala215Val(GCC $\rightarrow$ GTC) (Table 4).

Table 4

The MICs of LZD and $r p / C$, rp/D and 23srRNA mutations against $M$. fortuitum isolates.

\begin{tabular}{|c|c|c|c|c|}
\hline $\begin{array}{l}\text { MIC of LZD } \\
(\mu \mathrm{g} / \mathrm{ml})\end{array}$ & $\begin{array}{l}\text { Species } \\
\text { (NO.) }\end{array}$ & RplC & RplD & 23SrRNA \\
\hline \multirow[t]{2}{*}{4} & 1 & - & $\begin{array}{l}\text { Ala146Gly + Thr147Ser + Val156lle + Ala161Thr+ } \\
\text { Lys167Arg + Ser207Ala+ }\end{array}$ & \\
\hline & & & Glu212Gly + Val213Ala + Ala215Val (1) & \\
\hline \multirow[t]{3}{*}{8} & 2 & & $\begin{array}{l}\text { Ala146Gly + Thr147Ser + Val156lle + Ala161Thr + } \\
\text { Lys167Arg + Ser207Ala+ }\end{array}$ & \\
\hline & & & Glu212Gly + Val213Ala + Ala215Val (1) & \\
\hline & & & WT & $\mathrm{C} 1944 \mathrm{~T}(1)$ \\
\hline \multirow[t]{2}{*}{16} & 9 & & $\begin{array}{l}\text { Ala146Gly + Thr147Ser + Val156lle + Ala161Thr+ } \\
\text { Lys167Arg + Ser207Ala+ }\end{array}$ & \\
\hline & & & Glu212Gly + Val213Ala + Ala215Val (8) & \\
\hline \multirow[t]{3}{*}{32} & 7 & & $\begin{array}{l}\text { Ala161 Thr + Lys167Arg + Ser207Ala + Glu212Gly + } \\
\text { Val213Ala + Ala215Val(1) }\end{array}$ & A2090T(1) \\
\hline & & & $\begin{array}{l}\text { Ala146Gly + Thr147Ser + Val156lle + Ala161Thr+ } \\
\text { Lys167Arg + Ser207Ala+ }\end{array}$ & \\
\hline & & & Glu212Gly + Val213Ala + Ala215Val (5) & \\
\hline \multirow[t]{2}{*}{$>32$} & 6 & & $\begin{array}{l}\text { Ala146Gly + Thr147Ser + Val156lle + Ala161Thr + } \\
\text { Lys167Arg + Ser207Ala+ }\end{array}$ & \\
\hline & & & Glu212Gly + Val213Ala + Ala215Val (6) & \\
\hline
\end{tabular}

\section{Structural mapping of clinical mutants}

For M. massiliense isolates, Ala177Pro in RpID was detected in 12 isolates, both in LZD susceptible and resistant isolates with $\mathrm{MIC} \geq 2 \mu \mathrm{g} / \mathrm{mL}$. To gain an insight into the functional relevance of RplC and RpID mutation, multiple sequences alignment of RpIC and RpID homologues from different mycobacterial species were performed (Figure S1 and S2). The protein sequence of RpIC and RpID in different mycobacterial species are highly conserved. In addition, we used $M$. tuberculosis RpID structure as a 
model to map M. massiliense RpID mutation(PDB ID:5V7Q) (Fig. 5B). The structure shows that Ala177 is located in a high variable region between $\beta 3$ and $\eta 2$ and is far from the LZD binding site which indicates that this mutation may not be related to LZD resistance(Fig. 3B). Next, we mapped the 23SrRNA functional mutations of $M$. abscessus, $M$. massiliense and $M$. fortutium. The results showed that except A2271 in M. massiliense, the other mutations including G2582, A2625 and T2650 were far from the catalytic center (Fig. 3C).

\section{Discussion}

The treatment of RGM infection is often very difficult because of their higher drug resistance rate than SGM and unavailability of highly potent drugs against them in vitro. $M$. abscessus complex and $M$. fortuitum are two most prevalent RGM species around the world. Infections due to $M$. abscessus carry a poor prognosis since this RGM is, for all the correct reasons, considered an "antibiotic nightmare"(16). Thus, identifying drugs that could work potently against $M$. abscessus is a priority. M. massiliense is a species that originally splits from $M$. abscessus but it is located closely in the phylogenetic tree (17). The treatment response rates to clarithromycin-based antibiotic therapy are much higher in patients with $M$. massiliense than patients with $M$. abscessus lung disease (18). M. fortuitum is the main RGM responsible for extra-pulmonary disease, especially in cutaneous and plastic surgery-related infections(19). In contrast to M. abscessus, M. fortuitum infection has better prognosis due to some available effective drugs(20). However, its emerging drug resistance highlights the need for new and effective drugs(20-22). Several studies have verified the efficacy of LZD in MDR-TB or even in XDR-TB treatment $(9,13,23)$. A few studies also proved its antibacterial activity against NTM species either in vitro or in vivo $(24,25)$. As a novel oxazolidinone prodrug, TZD exhibited greater potency than LZD against $M$. tuberculosis $(6,26)$ as well as against $\operatorname{NTM}(27,28)$. Limited studies or no study has been performed to evaluate the efficacy of SZD and DZD against NTM species (28), whereas only a few studies provided preliminarily assessment of their potential usage in TB $(14,29,30)$. In this study, we evaluated the efficacies of four oxazolidinones against the reference and clinical isolates of RGM to gain insights into their potential use for specific RGM species.

As new drugs, well recognized susceptibility testing methods for TZD, SZD and DTD have not been developed and the breakpoints to define drug resistance for them have never been discussed yet. Therefore, the MIC data of different RGM species against oxazolidinones still remain scarce. In this study, the four oxazolidinones exhibited promising activities in vitro against the recruited RGM reference stains. The absolute majority of species had MICs below $8 \mu \mathrm{g} / \mathrm{mL}$ against the four drugs. However, different species presented non-uniform susceptibility patterns. The MIC distributions of $M$. massiliense had similar tendency to the M. abscessus, but the MICs of TZD were obviously higher than M. abscessus. Brown-Elliott et al demonstrated $M$. abscessus is more resistant to TZD, compared with M. massiliense with 2-fold lower $\mathrm{MIC}_{90}$ and $\mathrm{MIC}_{50}$ (28). However, Ruiz et al showed the $\mathrm{MIC}_{50}$ of $M$. massiliense against tedizolid was two fold higher than that of $M$. abscessus (6). The main reason for the this discrepancy is sampling error, the constituent ratio of $M$. abscessus and $M$. massiliense was different among 
studies.Furthmore, the resistant profile of stains isolate from different countries may be not exactly the same.In comparison with other oxazolidinones, the MIC values for TZD were the lowest for both $M$. abscessus and M. massiliense. Previous studies, including 170 isolates of RGM, showed equivalent or lower (1 to 8 fold) $\mathrm{MIC}_{50}$ and $\mathrm{MIC}_{90}$ values for TZD in contrast with LZD(28). Furthermore, TZD harbors several advantages over LZD in terms of tolerability, safety, dosing frequency, and treatment duration(31). Only a few studies have reported the clinical use of TZD for the treatment of NTM infections. Our results indicated that its usage seems reasonable for the treatment of infection caused by M. abscessus and $M$. massiliense. Among the 25 tested M. fortuitum isolates in our study, 22 (22/25) strains had MICs of LZD at $\geq 16 \mathrm{mg} / \mathrm{L}$. Based on the CLSI criteria, these strains could be categorized as intermediate resistant or resistant strains, $52 \%(13 / 25)$ of them belong to resistant strains. Using the cutoff value of LZD as the tentative breakpoints, the susceptibility rate of $M$. fortuitum against TZD, SZD and DZD were $20 \%(5 / 25)$, $12 \%$ (3/25), 76\% (19/25), respectively. DZD exhibited the best antimicrobial activity against the $M$. fortuitum. However, whether this in vitro outcome reflects the in vivo efficacy or not, requires further investigation.

A major limitation of this study was that no recommended breakpoint of different NTM species against TZD, SZD or DZD had been proposed previously. Besides in vitro MIC distributions, the breakpoint determination also correlates with clinical treatment response and pharmacokinetic/pharmacodynamics (PK/PD) data including drug dose. The clinical trials on these new oxazolidinones are either unavailable or very limited. A few studies have been performed on the pharmacokinetic analysis of these drugs. Generally, all the drugs were well-tolerated, and the $C_{\max }$ were highly dose-dependent. Recently, Choi et al demonstrated that, after multiple doses of TZD up to $1200 \mathrm{mg}$ twice daily for 21 days, the peak serum concentration was $16.3 \mu \mathrm{g} / \mathrm{mL}$, which is comparable with peak serum concentrations of LZD $=12.5$ $\mu \mathrm{g} / \mathrm{mL}$ at the dosage of $300 \mathrm{mg}$ twice daily $(15,32)$. In another study, a single $800 \mathrm{mg}$ dose of DZD under fasting condition acquired $\mathrm{C}_{\max }$ at $11.74 \mu \mathrm{g} / \mathrm{mL}(33)$. STD presented superior efficacy than LZD against experimental murine model of tuberculosis. The $C_{\max }$ of its major metabolite PNU-101603, which contributes to its activity, was $6.46 \mu \mathrm{g} / \mathrm{mL}$ at given a dose of $1200 \mathrm{mg}$ QD (40). However, since the optimal dosage of these next-generation oxazolidinones is still under investigation, the appropriate breakpoints for the susceptibility definition of these drugs remain beyond known.

LZD works by binding to the peptidyl transferase center of the $50 \mathrm{~S}$ ribosomal subunit, which is composed of $5 S$ and 23S rRNAs and 36 riboproteins (L1 through L36)(34). Recently, the Cryo-EM structure of the large ribosomal subunit from $M$. tuberculosis bound with a potent LZD analog (LZD-114) was determined(35). LZD-114 is similar to LZD in C ring but different in A and $B$ ring, in that it lacks a fluorine group in the B-ring while the original morpholine ring is replaced by a thiazole ring in the A-ring (Fig. $3 \mathrm{~A})$. The LZD-114 is also bound in the same pocket and in a similar orientation to LZD in other species (36, 37). The structure showed that $r p / C$ encoded ribosomal protein $L 3$ and $r p / D$ encoded ribosomal protein $L 4$ bound directly to 23S ribosomal RNA and was placed relatively close to the LZD binding site on the ribosomes, suggesting that the mechanism for reduced susceptibility may include structural perturbation of the LZD binding site (PDB:5V7Q). Furthermore, previous studies demonstrated that mutations in $r p / C$ 
and $r p / D$ could lead to LZD resistance in $M$. tuberculosis $(12,38)$. However, there is no non-synonymous mutation in $r p / C$ against the tested RGM. A1 la77Pro mutation was detected in $r p / D$ which is located in variable site and is far away from LZD-binding site, as shown by the sequence alignment. Except A2271G mutation in 23SrRNA in M. massiliense that was closer to binding site of LZD, other mutations are far from the LZD-binding site. Our results combining MIC test, gene mutation and structure based analysis showed there was no obvious correlation between riboproteins mutations $(r p / C$ and $r p / D)$ and LZD resistance against tested RGM species. Mutations located in the LZD binding site may cause LZD resistant. Hence, $r p / C, r p / D$ and 23srRNA homologues might not be the only target for LZD to explore its bacteriostatic activity.

In conclusion, this study demonstrated that oxazolidinones have good in vitro activities against the overwhelming majority of RGM species. The efficacies of the four oxazolidinones were variable against different species. TZD showed strongest antimicrobial activity against $M$. abscessus and $M$. massiliense, while DZD owned the strongest activity against $M$. fortuitum. The data provided important insights into the possible clinical applications of oxazolidinones to treating RGM infections(39).

\section{Material And Methods}

\section{Ethics statement}

As the study only concerned laboratory testing of mycobacteria without the direct involvement of human subjects, ethics approval was not sought.

\section{Reference strains and clinical isolates}

The mycobacterial reference strains stored in the Bio-bank in Beijing Chest Hospital (Beijing, China) were tested against LZD, TZD, SZD and DZD in vitro, including 32 RGM species. These reference strains were obtained either from the American Type Culture Collection (ATCC) or from the German Collection of Microorganisms (DSM). The species constitution of these reference strains is listed in Table 1. M. massiliense reference strain was not included due to its absence in our stock. One-hundred fifteen isolates of RGM were recruited in Beijing chest hospital from 2016 to 2018 that included $49 \mathrm{M}$. abscessus, $35 \mathrm{M}$. massiliense, $25 \mathrm{M}$. fortuitum. The species constitution of the remaining 6 isolates is presented in Table 2.

All of the 115 RGM clinical strains were isolated from tuberculosis suspected patients. The strains were classified as RGM preliminarily with p-nitrobenzoic acid containing medium, and then were identified by gene sequencing as indicated for each species by 16S rRNA, hsp65, rpoB, 16-23S rRNA internal transcribed spacer sequencing (40). All the isolates were stored at $-80^{\circ} \mathrm{C}$ and sub-cultured on LJ medium before performing drug susceptibility test. 


\section{Minimal inhibitory concentration (MIC) testing}

TZD phosphate and LZD were purchased from Toronto Research Chemicals and Sigma-aldrich, respectively. SZD and DZD were purchased from Shanghai Biochempartner Co., Ltd (Shanghai, China) and JHK BioPharma, respectively. Oxazolidinones were dissolved in dimethyl sulfoxide (DMSO). Stock solutions were aseptically prepared at concentrations of $2.56 \mathrm{mg} / \mathrm{mL}$. Broth microdilution method was performed according to the guidelines of Clinical and Laboratory Standards Institute (CLSI)(41). Cationadjusted Mueller-Hinton broth (CAMHB) was used for MIC test. The inoculum was prepared with fresh culture grown on Lowenstein-Jensen medium. The broth microdilution format was set up as 2-fold dilution $邓$ the concentrations of all the tested drugs ranged from $0.063 \mu \mathrm{g} / \mathrm{mL}$ to $32 \mu \mathrm{g} / \mathrm{mL}$. Briefly, a bacterial suspension of $0.5 \mathrm{McF}$ arland standard was prepared, and then diluted and inoculated into 96well microtiter plate to achieve final bacterial load at $10^{5}$ colony forming unit (CFU) per well. Plates were then incubated at $37^{\circ} \mathrm{C}$ for 3 days for RGM. $70 \mu \mathrm{L}$ solution containing $20 \mu \mathrm{L}$ AlamarBlue (Bio-rad) and $50 \mu \mathrm{L}$ Tween80 (5\%) was added to each well and incubated for $24 \mathrm{~h}$ at $37^{\circ} \mathrm{C}$ before assessing color development. A change from blue to pink or purple indicated bacterial growth (42). The MIC was defined as the lowest concentration of antibiotic that prevented a color change from blue to pink.

The breakpoint of LZD was adopted from the CLSI document M24-A2 (susceptible: $\leq 8 \mathrm{mg} / \mathrm{L}$; intermediate resistant: $16 \mathrm{mg} / \mathrm{L}$; resistant: $\geq 32 \mathrm{mg} / \mathrm{L}$ ) (43). Since no well-recognized breakpoint has been proposed for TZD, SZD or DZD, a preliminary data analysis was performed for them referring the breakpoint of LZD.

\section{Mutations potentially conferring oxazolidinones resistance and protein alignment}

Sequencing of PCR products was performed using the Sanger method with primers designed to be specific for rpIC, rpID and 23S rRNA. We used previously described primers for $23 S$ rRNA(44), and designed new primers for rpIC, rpID sequencing. The primers used in this study are listed in Table S1 in the supplemental material and were synthesized by Tsingke Biotech Co. (Beijing, China). The rpIC and rpID gene of the reference strains of three RGM species plus M. tuberculosis were also sequenced, mutation was defined in contrast with the sequences of the reference strains. The sequences of $M$. massiliense were adopted from website for alignment. The amplification products were sequenced by Tsingke Company (Beijing, China). Multiple sequence alignment of the homologous proteins was performed using the Clustal Omega software. Structure-based multiple sequence alignment was performed with ESPript 3 based on the crystal structure of RpIC and RpID protein of M .tuberculosis from the following URL:http://espript.ibcp.fr/ESPript/ESPript/.

\section{Quality control.}


The MIC for quality control strains was determined using each lot of the prepared microtiter plates, and the results for LZD were within the expected range.

\section{Declarations}

\section{Consent for publication}

Not applicable.

\section{Availability of data and material}

The datasets generated during and/or analysed during the current study are not publicly available due [REASON WHY DATA ARE NOT PUBLIC] but are available from the corresponding author on reasonable request.

\section{ACKNOWLEDGEMENT}

This study was supported by research funding from the Infectious Diseases Special Project, Ministry of Health of China (2018ZX10302302-004-005, 2018ZX10201301302-004.) the Natural Science Fund of China (81672065 81802057$)$, Beijing Municipal Administration of Hospitals Clinical Medicine Development of Special Funding Support\ZYLX201824囚and Beijing Municipal Administration of Hospitals' Ascent Plan (DFL20181602).

\section{Competing interests}

The authors declare that they have no conflict of interest.

\section{Author's contributions}

Hairong Huang and Xia Yu designed the study and wrote the paper;Shuan Wen, Xiaopan Gao,Weijie Zhao ,Fengmin Huo,Guanglu Jiang, Lingling Dong, Liping Zhao and Fen Wang performed experiments.Hairong Huang,Xia Yu and Shuan Wen analyzed the data. All authors reviewed the results and approved the final version of the manuscript.

\section{References}

1. Cowman S, van Ingen J, Griffith DE, Loebinger MR. 2019. Non-tuberculous mycobacterial pulmonary disease. Eur Respir J 54. 
2. Santin M, Barrabeig I, Malchair P, Gonzalez-Luquero L, Benitez MA, Sabria J, Palau-Benavent M, Canete C, Lloret-Queralto JA, Grijota-Camino MD, Dorca J, Alcaide F. 2018. Pulmonary Infections with Nontuberculous Mycobacteria, Catalonia, Spain, 1994-2014. Emerg Infect Dis 24:1091-1094.

3. Lin C, Russell C, Soll B, Chow D, Bamrah S, Brostrom R, Kim W, Scott J, Bankowski MJ. 2018. Increasing Prevalence of Nontuberculous Mycobacteria in Respiratory Specimens from US-Affiliated Pacific Island Jurisdictions(1). Emerg Infect Dis 24:485-491.

4. Brode SK, Marchand-Austin A, Jamieson FB, Marras TK. 2017. Pulmonary versus Nonpulmonary Nontuberculous Mycobacteria, Ontario, Canada. Emerg Infect Dis 23:1898-1901.

5. Yu X, Liu P, Liu G, Zhao L, Hu Y, Wei G, Luo J, Huang H. 2016. The prevalence of non-tuberculous mycobacterial infections in mainland China: Systematic review and meta-analysis. J Infect 73:558567.

6. Ruiz P, Causse M, Vaquero M, Casal M. 2019. In Vitro Activity of Tedizolid against Mycobacterium tuberculosis. Antimicrob Agents Chemother 63.

7. Compain F, Soroka D, Heym B, Gaillard JL, Herrmann JL, Dorchene D, Arthur M, Dubee V. 2018. In vitro activity of tedizolid against the Mycobacterium abscessus complex. Diagn Microbiol Infect Dis 90:186-189.

8. Celdran A, Esteban J, Manas J, Granizo JJ. 2007. Wound infections due to Mycobacterium fortuitum after polypropylene mesh inguinal hernia repair. J Hosp Infect 66:374-7.

9. Singh B, Cocker D, Ryan H, Sloan DJ. 2019. Linezolid for drug-resistant pulmonary tuberculosis. Cochrane Database Syst Rev 3:CD012836.

10. Bilgin H, Tukenmez-Tigen E. 2019. Linezolid for drug-susceptible tuberculosis. Lancet Infect Dis 19:357.

11. Bolhuis MS, Akkerman OW, Sturkenboom MGG, Ghimire S, Srivastava S, Gumbo T, Alffenaar JC. 2018. Linezolid-based Regimens for Multidrug-resistant Tuberculosis (TB): A Systematic Review to Establish or Revise the Current Recommended Dose for TB Treatment. Clin Infect Dis 67:S327-S335.

12. Zong Z, Jing W, Shi J, Wen S, Zhang T, Huo F, Shang Y, Liang Q, Huang H, Pang Y. 2018. Comparison of In Vitro Activity and MIC Distributions between the Novel Oxazolidinone Delpazolid and Linezolid against Multidrug-Resistant and Extensively Drug-Resistant Mycobacterium tuberculosis in China. Antimicrob Agents Chemother 62.

13. Lee M, Lee J, Carroll MW, Choi H, Min S, Song T, Via LE, Goldfeder LC, Kang E, Jin B, Park H, Kwak H, Kim H, Jeon HS, Jeong I, Joh JS, Chen RY, Olivier KN, Shaw PA, Follmann D, Song SD, Lee JK, Lee D, Kim CT, Dartois V, Park SK, Cho SN, Barry CE, 3rd. 2012. Linezolid for treatment of chronic extensively drug-resistant tuberculosis. N Engl J Med 367:1508-18.

14. Wallis RS, Dawson R, Friedrich So, Venter A, Paige D, Zhu T, Silvia A, Gobey J, Ellery C, Zhang Y, Eisenach K, Miller P, Diacon AH. 2014. Mycobactericidal activity of sutezolid (PNU-100480) in sputum (EBA) and blood (WBA) of patients with pulmonary tuberculosis. PLoS One 9:e94462.

15. Cho YS, Lim HS, Lee SH, Cho YL, Nam HS, Bae KS. 2018. Pharmacokinetics, Pharmacodynamics, and Tolerability of Single-Dose Oral LCB01-0371, a Novel Oxazolidinone with Broad-Spectrum 
Activity, in Healthy Volunteers. Antimicrob Agents Chemother 62.

16. Nessar R, Cambau E, Reyrat JM, Murray A, Gicquel B. 2012. Mycobacterium abscessus: a new antibiotic nightmare. J Antimicrob Chemother 67:810-8.

17. Adekambi T, Reynaud-Gaubert M, Greub G, Gevaudan MJ, La Scola B, Raoult D, Drancourt M. 2004. Amoebal coculture of "Mycobacterium massiliense" sp. nov. from the sputum of a patient with hemoptoic pneumonia. J Clin Microbiol 42:5493-501.

18. Koh WJ, Jeon K, Lee NY, Kim BJ, Kook YH, Lee SH, Park YK, Kim CK, Shin SJ, Huitt GA, Daley CL, Kwon OJ. 2011. Clinical significance of differentiation of Mycobacterium massiliense from Mycobacterium abscessus. Am J Respir Crit Care Med 183:405-10.

19. Brown-Elliott BA, Wallace RJ, Jr. 2002. Clinical and taxonomic status of pathogenic nonpigmented or late-pigmenting rapidly growing mycobacteria. Clin Microbiol Rev 15:716-46.

20. Shen Y, Wang X, Jin J, Wu J, Zhang X, Chen J, Zhang W. 2018. In Vitro Susceptibility of Mycobacterium abscessus and Mycobacterium fortuitum Isolates to 30 Antibiotics. Biomed Res Int 2018:4902941.

21. Aono A, Morimoto K, Chikamatsu K, Yamada H, Igarashi Y, Murase Y, Takaki A, Mitarai S. 2019. Antimicrobial susceptibility testing of Mycobacteroides (Mycobacterium) abscessus complex, Mycolicibacterium (Mycobacterium) fortuitum, and Mycobacteroides (Mycobacterium) chelonae. J Infect Chemother 25:117-123.

22. Sparks R, Khatami A. 2014. Mycobacterium fortuitum Complex Skin Infection in a Healthy Adolescent. Infect Disord Drug Targets 14:168-71.

23. Hashemian SMR, Farhadi T, Ganjparvar M. 2018. Linezolid: a review of its properties, function, and use in critical care. Drug Des Devel Ther 12:1759-1767.

24. Cavusoglu C, Soyler I, Akinci P. 2007. Activities of Linezolid against nontuberculous mycobacteria. New Microbiol 30:411-4.

25. Brown-Elliott BA, Crist CJ, Mann LB, Wilson RW, Wallace RJ, Jr. 2003. In vitro activity of linezolid against slowly growing nontuberculous Mycobacteria. Antimicrob Agents Chemother 47:1736-8.

26. Prokocimer P, Bien P, Surber J, Mehra P, DeAnda C, Bulitta JB, Corey GR. 2011. Phase 2, randomized, double-blind, dose-ranging study evaluating the safety, tolerability, population pharmacokinetics, and efficacy of oral torezolid phosphate in patients with complicated skin and skin structure infections. Antimicrob Agents Chemother 55:583-92.

27. Vera-Cabrera L, Brown-Elliott BA, Wallace RJ, Jr., Ocampo-Candiani J, Welsh O, Choi SH, MolinaTorres CA. 2006. In vitro activities of the novel oxazolidinones DA-7867 and DA-7157 against rapidly and slowly growing mycobacteria. Antimicrob Agents Chemother 50:4027-9.

28. Brown-Elliott BA, Wallace RJ, Jr. 2017. In Vitro Susceptibility Testing of Tedizolid against Nontuberculous Mycobacteria. J Clin Microbiol 55:1747-1754.

29. Zong Z, Jing W, Shi J, Wen S, Zhang T, Huo F, Shang Y, Liang Q, Huang H, Pang Y. 2018. Comparison of in vitro activity and MIC distributions between the novel oxazolidinone delpazolid and linezolid 
against multidrug-resistant and extensively drug-resistant Mycobacterium tuberculosis in China. Antimicrob Agents Chemother doi:10.1128/aac.00165-18.

30. McNeil MB, Dennison DD, Shelton CD, Parish T. 2017. In Vitro Isolation and Characterization of Oxazolidinone-Resistant Mycobacterium tuberculosis. Antimicrob Agents Chemother 61.

31. Flanagan S, Minassian SL, Prokocimer P. 2018. Pharmacokinetics of Tedizolid and Pseudoephedrine Administered Alone or in Combination in Healthy Volunteers. J Clin Med 7.

32. Choi Y, Lee SW, Kim A, Jang K, Nam H, Cho YL, Yu KS, Jang IJ, Chung JY. 2018. Safety, tolerability and pharmacokinetics of 21 day multiple oral administration of a new oxazolidinone antibiotic, LCB01-0371, in healthy male subjects. J Antimicrob Chemother 73:183-190.

33. Sunwoo J, Kim YK, Choi Y, Yu KS, Nam H, Cho YL, Yoon S, Chung JY. 2018. Effect of food on the pharmacokinetic characteristics of a single oral dose of LCB01-0371, a novel oxazolidinone antibiotic. Drug Des Devel Ther 12:1707-1714.

34. Dong W, Chochua S, McGee L, Jackson D, Klugman KP, Vidal JE. 2014. Mutations within the rpID Gene of Linezolid-Nonsusceptible Streptococcus pneumoniae Strains Isolated in the United States. Antimicrob Agents Chemother 58:2459-62.

35. Yang K, Chang JY, Cui Z, Li X, Meng R, Duan L, Thongchol J, Jakana J, Huwe CM, Sacchettini JC, Zhang J. 2017. Structural insights into species-specific features of the ribosome from the human pathogen Mycobacterium tuberculosis. Nucleic Acids Res 45:10884-10894.

36. Eyal Z, Matzov D, Krupkin M, Wekselman I, Paukner S, Zimmerman E, Rozenberg H, Bashan A, Yonath A. 2015. Structural insights into species-specific features of the ribosome from the pathogen Staphylococcus aureus. Proc Natl Acad Sci U S A 112:E5805-14.

37. Ippolito JA, Kanyo ZF, Wang D, Franceschi FJ, Moore PB, Steitz TA, Duffy EM. 2008. Crystal structure of the oxazolidinone antibiotic linezolid bound to the 50S ribosomal subunit. J Med Chem 51:3353-6.

38. Beckert P, Hillemann D, Kohl TA, Kalinowski J, Richter E, Niemann S, Feuerriegel S. 2012. rpIC T460C identified as a dominant mutation in linezolid-resistant Mycobacterium tuberculosis strains. Antimicrob Agents Chemother 56:2743-5.

39. Wen S, Gao X, Zhao W, Huo F, Jiang G, Dong L, Zhao L, Wang F, Yu X, Huang H. 2020. <em>In Vitro</em> efficacy comparison of linezolid, tedizolid, sutezolid and delpazolid against rapid growing Mycobacteria isolated in Beijing, China. bioRxiv.

40. Pang YA-Ohoo, Zheng H, Tan Y, Song Y, Zhao Y. 2017. In Vitro Activity of Bedaquiline against Nontuberculous Mycobacteria in China. LID - e02627-16 [pii] LID - 10.1128/AAC.02627-16 [doi]. Antimicrob Agents Chemother 61.

41. Clinical and Laboratory Standards Institute 2011. Susceptibility testing of mycobacteria, nocardia, and other aerobic actinomycetes; approved standard, 2nd ed; CLSI document M24-A2, Wayne, PA.

42. Coeck N, de Jong BC, Diels M, de Rijk P, Ardizzoni E, Van Deun A, Rigouts L. 2016. Correlation of different phenotypic drug susceptibility testing methods for four fluoroquinolones in Mycobacterium tuberculosis. J Antimicrob Chemother 71:1233-40. 
43. Clinical and Laboratory Standards Institute2011. Susceptibility testing of mycobacteria, nocardiae, and other aerobic actinomycetes; approved standard, 2nd ed. CLSI document M24-A2., Wayne, PA.

44. Kim SY, Jhun BW, Moon SM, Jeon K, Kwon OJ, Huh HJ, Lee NY, Shin SJ, Daley CL, Koh WJ. 2019. Genetic mutations in linezolid-resistant Mycobacterium avium complex and Mycobacterium abscessus clinical isolates. Diagn Microbiol Infect Dis 94:38-40.

\section{Figures}

\section{Figure 1}
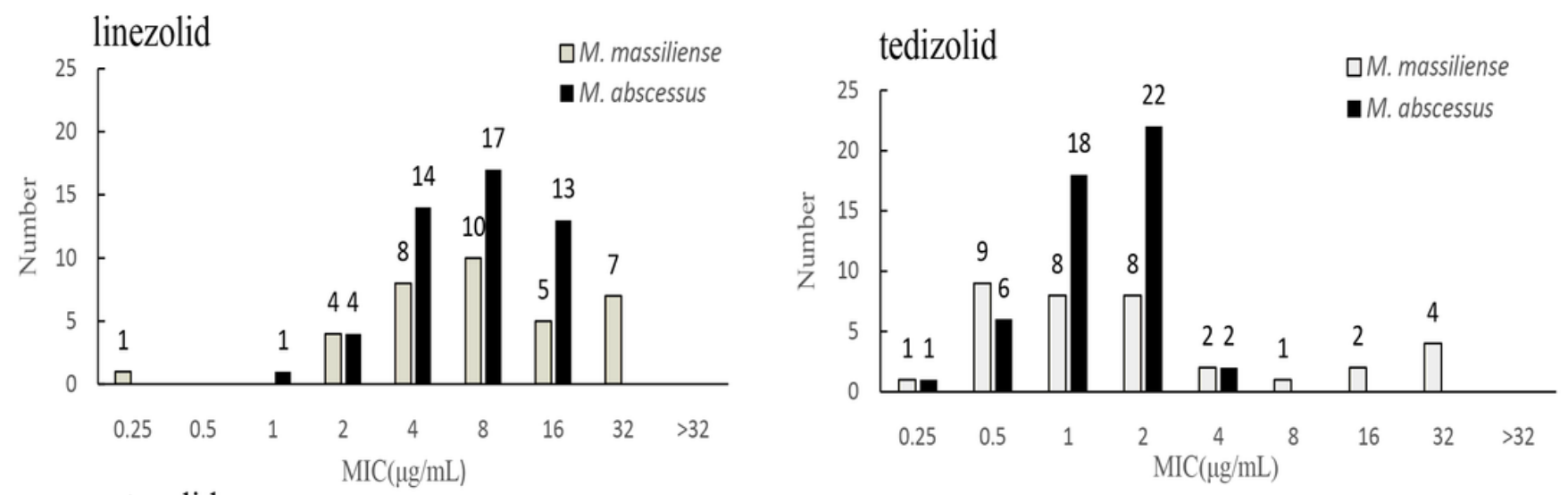

sutezolid

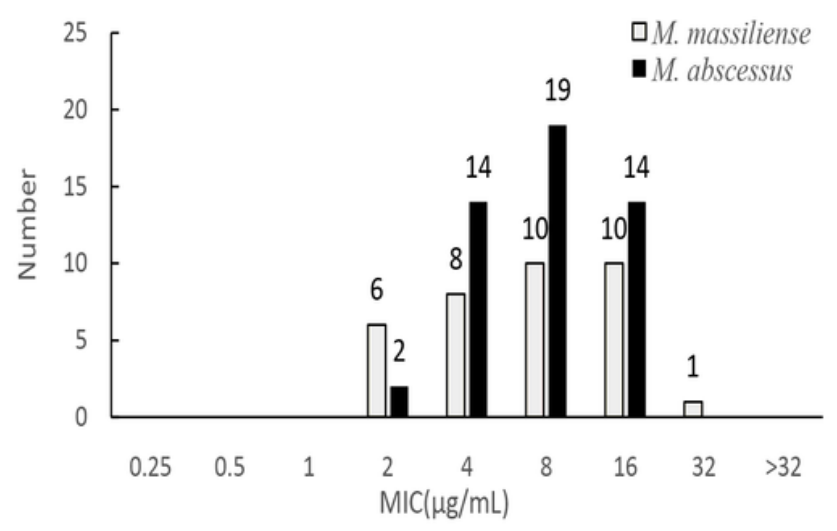

delpazolid

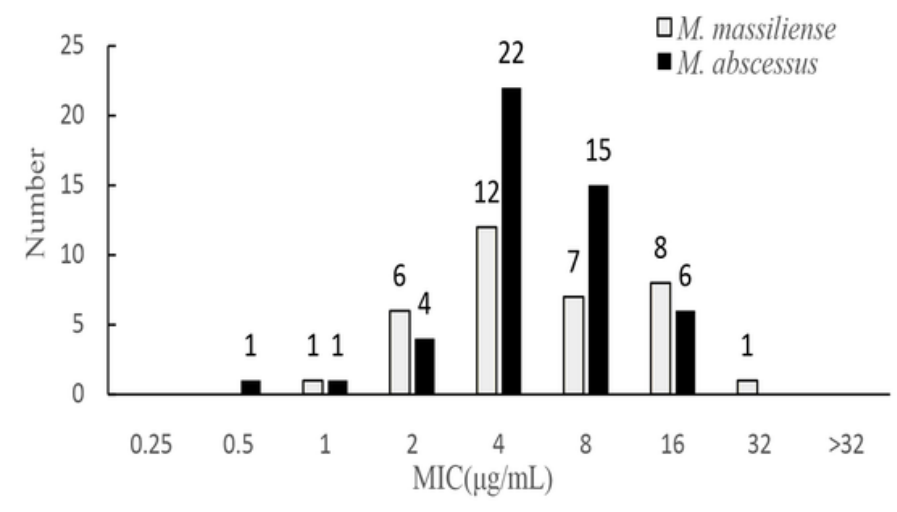

Figure 1

The MIC distributions of M. abscessus and M. massiliense against LZD, TZD, SZD and DZD. 
Figure 2
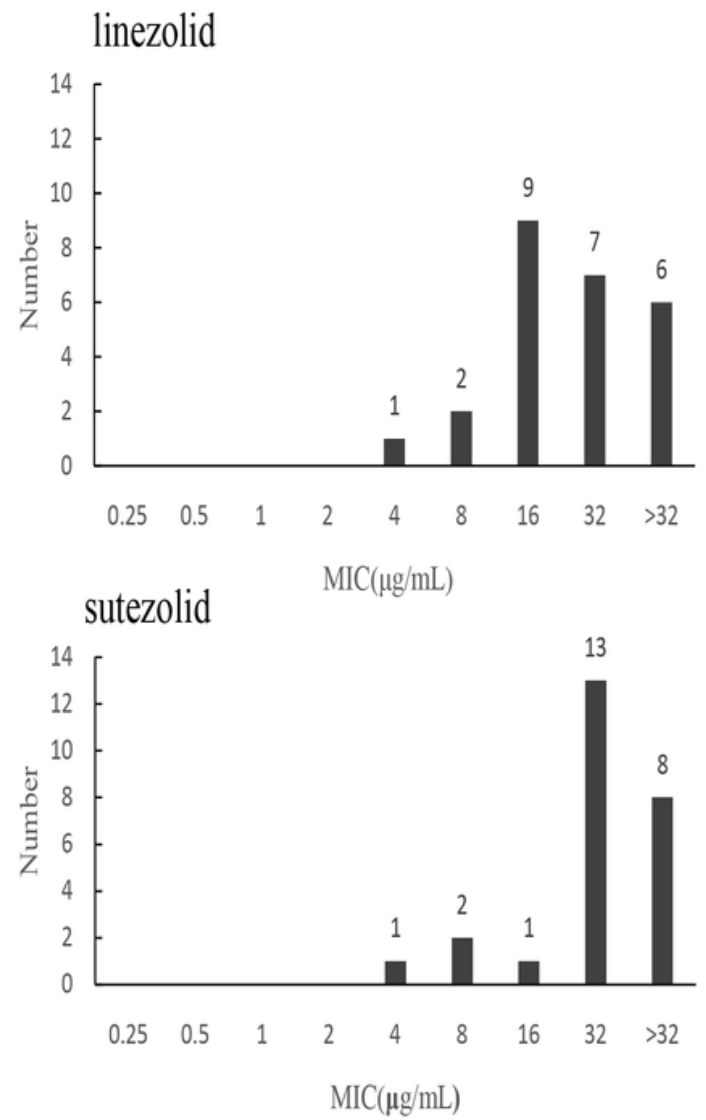
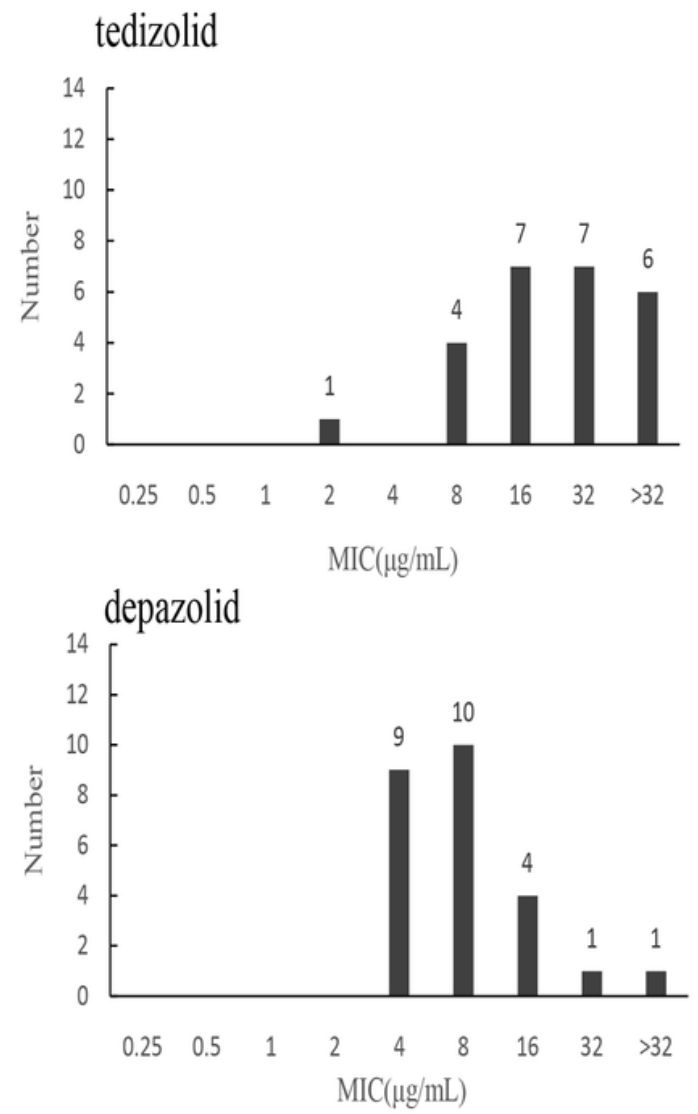

Figure 2

The MIC distributions of M. fortuitum against LZD,TZD, SZD and DZD. 


\section{Figure.3}

A

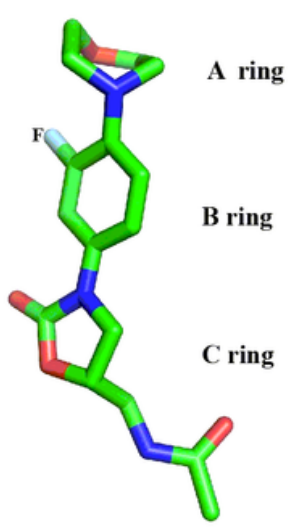

LZD
C

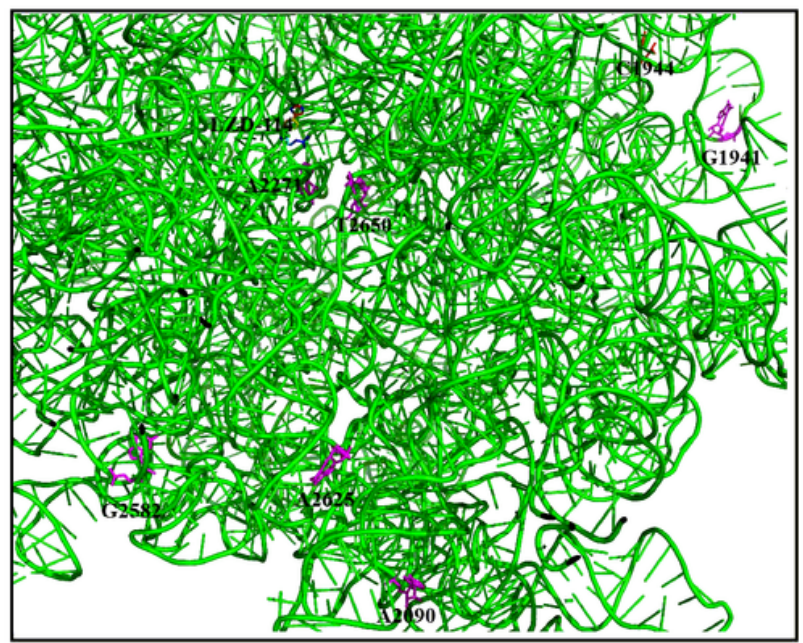

LZD-114

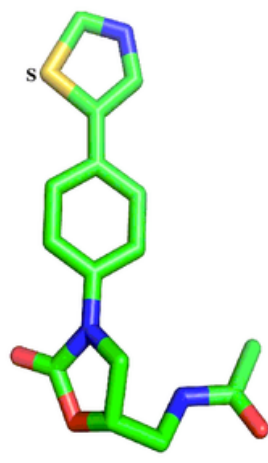

\section{B}

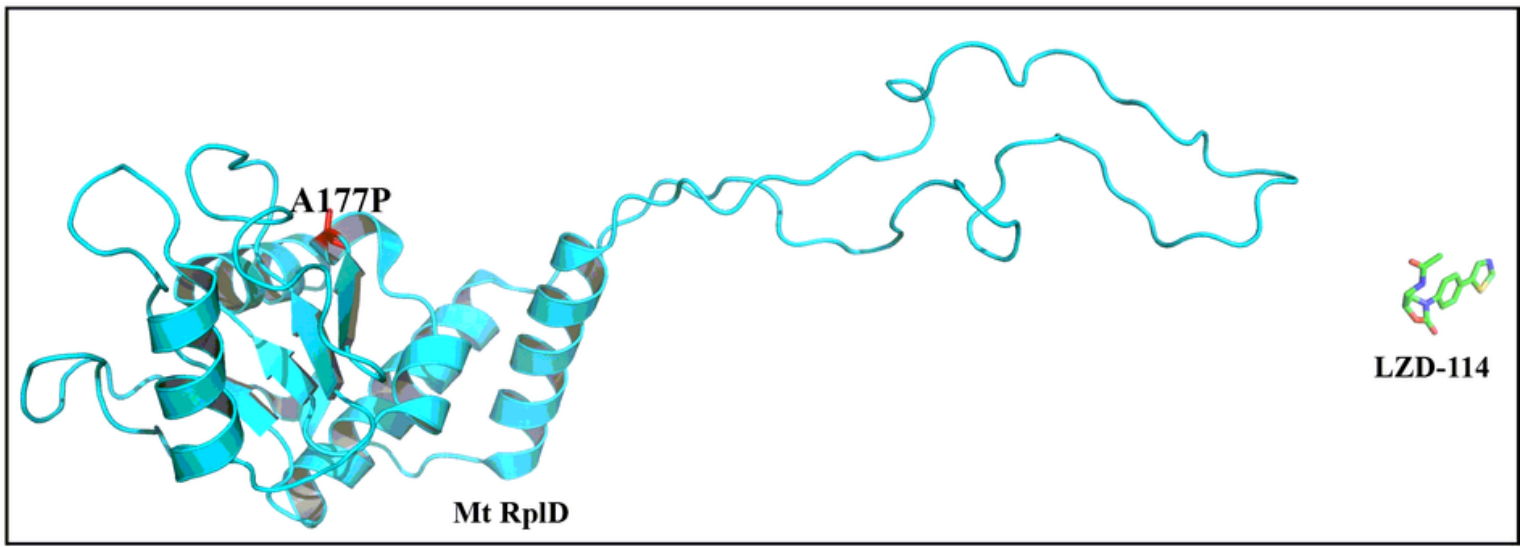

\section{Figure 3}

The structure of the ribosomal 23SrRNA and rpID. (A)The structure of LZD and its analog LZD-114.

(B)The structure of rpID and Ala177Pro mutations detected in M.massiliense isolated highlighted in red.

(C) The structure of ribosomal 23SrRNA and mutations detected in tested RGM clinical isolates were highlighted in red.

\section{Supplementary Files}

This is a list of supplementary files associated with this preprint. Click to download.

- TableS1.Primersetsusedfortargetgenesinthisstudy.docx 
- FigureS1.jpeg

- FigureS2rpID.jpeg 